\title{
Microbial community with predictive power can be a new non-invasive substitute for clinical and pathological identification of diabetic nephropathy
}

Jin Shang

Zhengzhou University First Affiliated Hospital

\section{Zhigang Ren}

Zhengzhou University First Affiliated Hospital

Ang Li

Zhengzhou University First Affiliated Hospital

\section{Ruixue Guo}

Zhengzhou University First Affiliated Hospital

Yiding Zhang

Zhengzhou University First Affiliated Hospital

\section{Hongyan Ren}

Shanghai Mobio Biomedical Technology Co., Ltd.

\section{Chao Liu}

Shanghai Mibio Biomedical Technology Co., Ltd

\section{Weifeng Zhang}

Zhengzhou University First Affiliated Hospital

\section{Fanliang Zhang}

Zhengzhou University First Affiliated Hospital

\section{Suying Ding}

Zhengzhou University First Affiliated Hospital

\section{Haiyu Wang}

Zhengzhou University First Affiliated Hospital

\section{Jing Xiao}

Zhengzhou University First Affiliated Hospital

zhanzheng Zhao ( $\square$ zhanzhengzhao@zzu.edu.cn )

Zhengzhou University First Affiliated Hospital https://orcid.org/0000-0001-6079-1631

\section{Research}

Keywords: gut microbiome, diabetic kidney disease, diabetic nephropathy, diabetic mellitus, membranous nephropathy, non-invasive test 
Posted Date: May 15th, 2020

DOI: https://doi.org/10.21203/rs.3.rs-28863/v1

License: (c) (i) This work is licensed under a Creative Commons Attribution 4.0 International License. Read Full License 


\section{Abstract \\ Background}

Diabetic nephropathy is characterized by increased incidence, deficient diagnostic methods and poor prognosis. New idea about altered gut microbiome associated with diagnosis and development of diabetic nephropathy remains to be verified. The major aim of our study is to relate fecal microbiome to clinically diagnosed diabetic kidney disease (DKD) or pathologically identified diabetic nephropathy (defined as DN) and further evaluate diagnosis potential of microbial markers for DKD/DN. We carried out 16S rRNA sequencing on a discovery cohort consisting of 352 patients ( $D K D=120, D M=$ diabetes mellitus $=92$, Con $=$ healthy controls $=140$ ) to identify microbial taxa and construct DKD classifier . Functional relevance and clinic correlation of microbiome changes were performed using PICRUSt and Spearman analysis, respectively. Independent 60 DKDs and 116 non-DKDs ( $D M=46, C o n=70)$ were used to validate the results. The same analysis was performed on $D K D$ pathologic subtypes $(D N=22, M N=$ membranous nephropathy $=22$ ).

\section{Results}

DKD/DM samples had a distinct microbiome signature with lower alpha-diversity and significantly different microbial composition compared with Con $(\mathrm{P}<0.001)$. Expansion of opportunistic pathogens (Peptostreptococcaceae_incertae_sedis, Clostridium_sensu_stricto_1, Streptococcus, Enterococcus, Erysipelotrichaceae_incertae_sedis), sulphate-reducing bacteria (Desulfovibrio) and depletion of bacteria producing short-chain fatty acids (SCFA) (Bacteroides, Faecalibacterium, Blautia and Roseburia) were major contributors to above differences. Interestingly, mucosa-associated bacteria including Akkermansia and Ruminococcus were also increased in DKD. The combination of 11 microbial markers could separate 120 DKDs from 232 non-DKDs with an area under curve (AUC) of $88.12 \%$. Correspondingly, diagnostic power of microbial biomarkers was evaluated in a validation cohort of 60 patients and 116 non-DKDs (AUC $=79.75 \%$ ). Besides DKD-related lipid and arginine metabolism, we also observed an increase of metabolism of aromatic amino acid in DM. Additionally, microbial comparison was carried out between pathologic subtypes of DKD, which could be used to distinguish DN from MN with $77.69 \%$ AUC.

\section{Conclusion}

Gut microbiome-related changes were associated with pathogenesis of DKD/DN; Microbiota-targeted markers could be an alternative test for DKD diagnosis and a non-invasive choice to differentiate DKD pathologic subtypes.

\section{Background:}


Diabetic kidney disease (DKD), one of the most prevalent complications of diabetes mellitus (DM) [1], is now the top reason of end-stage renal disease (ESRD) worldwide [2]. Diagnostic criteria of DKD mainly relies on clinical characteristics [3]. However, current evidence indicates that only approximately $25 \%$ of clinically diagnosed DKD will be confirmed by pathologic results [4]. The rest of these patients are just "renal disease complicated with DM" rather than real diabetic nephropathy (DN). In spite of being golden standard, renal biopsy is not feasible to many DKD patients considering their poor physical condition [5]. Treatment strategies targeting DKD appears to be limited in reducing incidence [6]. As a result, there is a dire need to explore new factors that may be involved in development of DKD, whereby find an alternative way for accurate diagnosing DKD and even substitute invasive renal biopsy.

Mounting evidence suggests that gut microbiota is considered to be linked and contribute to many complex diseases now. The microorganic community varies substantially between different health or disease conditions. It is generally believed that gut microbiota is associated with degrading indigestible carbohydrates [7], promoting host immune tolerance [8] and even facilitating the development certain diseases [9-11]. Accordingly, a large number of studies have indicated its potential medical ability in area of early diagnosis, prognostic prediction, treatment strategy and so on. In chronic kidney disease (CKD) and ESRD, gut microbiome interacts with impaired renal function through gut-kidney axis [12-15]. Gut microbiota also plays an important role in DM patients. It has been widely reported to be involved in insulin resistance and glucose metabolism-related disorder [10, 16]. In addition, diet adjustment targeting intestinal bacteria has great effects on improving hyperglycemia [17]. As for DN patients, Tao $\mathrm{S}$. and his colleagues have revealed the difference in gut microbiome between 14 biopsy-proven DNs and 14 DMs [18]. However, there is still a need to validate whether gut microbiome can help to distinguish DKD from DM in a larger scale and further to distinguish DN from other pathologic types, such as membranous nephropathy $(\mathrm{MN})$, superimposed with diabetes.

Here, we characterized compositional changes of gut microbiome in clinical-diagnosed patients with DKD, DM and healthy controls. Based on microbial comparison, we further constructed microbiomeassociated classifier for DKD and non-DKD in discovery phase and validation phase. Additionally, we compared gut microbiome of DN with $\mathrm{MN}$ and tried to construct pathologic sub-type classifier. To our knowledge, this is the first study focusing on gut microbiome alterations between different pathologic sub-types.

\section{Materials And Methods:}

\section{Study participants and samples collection}

All the patients enrolled were diagnosed as DKD or DM and were hospitalized in the first affiliated hospital of Zhengzhou University from October 2018 to October 2019. Fecal samples of age and gendermatched healthy controls were obtained at physical examination center. Our study followed the Helsinki declaration. Ethics Review Committee approved all experimental process. All individuals had rights to know and signed written informed consents before sample collection. 
Diagnostic criteria for DKD was at least 5 years history of diabetes complicated with repeated albuminuria (urinary protein/creatinine $\geq 30 \mathrm{mg} / \mathrm{g}$ ) or macro-protein urine [19]. Autoimmune disease, such as lupus nephritis, anti-neutrophil cytoplasmic antibodies (ANCA)-associated vasculitis, tumor and viral hepatitis were excluded. Forty-four DKD patients were subjected to renal biopsy and bacterial comparison was also performed on this pathological subgroup. $D N(n=22)$ referred to nephropathy secondary to diabetes, which was confirmed by pathology [20]. Some patients were clinically diagnosed as DKD at first. However, pathological result showed other types, like membranous nephropathy ( $\mathrm{MN}=$ 22). Healthy persons for Con group were screened in terms of following inclusion criteria: (1) normal urine test, serum albumin (Alb) and serum creatinine (Cr); (2) normal blood glucose and glycosylated hemoglobin $(\mathrm{GHb})$. One who reported basic diseases was excluded. Individuals with antibiotic application before sample collection were not included either.

We consecutively recruited 195 DKDs, 138 DMs and 210 healthy Controls. Owing to missed clinical information of 15 DKDs in subsequent process, final 528 participants were subjected to 16S rRNA gene sequencing. All the samples were freshly collected and immediately frozen at $-4^{\circ} \mathrm{C}$. Then they were transferred to a $-80^{\circ} \mathrm{C}$ environment within 2 hours for further analysis.

\section{Dna Extraction and 16s Metagenomic Sequencing}

Beads-beating method was used to lyse bacterial cell membrane. Bacterial DNA was extracted using E.Z.N.A. ${ }^{\circledR}$ Stool DNA Kit following previous study [21]. PCR amplification of 16S rRNA V3-V4 region genes was carried out to prepare gene library. Purified amplicons were used for sequencing on the Miseq platform (Illumina Inc., USA). All procedures were completed by Shanghai Mobio Biomedical Technology Co. Ltd. according to manufacturer's protocols. Details of DNA extraction, PCR amplification and primers design were stated in online Additional file 1.

\section{Otu Clustering and Annotation}

Chimeric sequences were removed using UPARSE (version $7.1 \mathrm{http} / / /$ drive5.com/uparse/). Then, we classified Operational Taxonomic Unit (OTUs) with 97\% similarity [22]. To analyze the phylogenetic affiliation of 16S rRNA gene sequence, we used RDP Classifier (http://rdp.cme.msu.edu/) against the Silva (SSU123) 16S rRNA database with confidence threshold of 70\%. Detailed sequence purification was listed in online Additional file 1.

\section{Bioinformatic Analysis}

Bacterial richness and diversity were estimated separately by Chao/Observed OTU and Shannon/Simpson indices in our study. Mann-Whitney $U$ test was used to compare OTU differences of two groups. Differences of three groups were compared using Kruskal-Wallis test. Bray-Curtis 
dissimilarity and (un)weighted UniFrac distances were calculated in QIIME to assess beta diversity. In addition, principal coordinate analysis (PCOA) and non-metric multidimensional scaling analysis (NMDS) were performed to aid interpretation of bacterial distribution among three groups. Correspondingly, we used permutational multivariate analysis of variance (PERMANOVA) and analysis of similarities (ANOSIM) to evaluate the statistical significance of differences among three groups. Significantly different genera in discovery cohort were determined using the linear discriminant analysis (LDA) effect size (LEfSe). Significant taxonomic distribution between DN and MN was shown with LDA values $>2.0$ and $\mathrm{P}$ value $<0.05$. Correlation between fecal microbiota and clinical variables was calculated by Spearman correlation analysis and showed in the pattern of heatmap. Phylogenetic Investigation of Communities by Reconstruction of Unobserved states (PICRUSt) was used to predict markedly enriched KEGG pathways in different disease status (LDA scores $>2.0$ and $P$ value $<0.05)$.

\section{Gut Microorganism-based Roc Construction}

Using the abundance profiles of OTU, five-fold cross-validation was used on random forest model to feature important OTUs (importance value >0.001) for classification of 120 DKDs and 232 non-DKDs (92 DMs and 140 Con). By five repeats of five-fold cross-validation, we got the minimum cross-validation (CV) error rate and defined this value as the cutoff. OTU set with a CV error rate less than cutoff and the minimum number was considered as the optimal set. Based on the optimal set, we performed receiver operating characteristic (ROC) curve construction for discovery cohort and validation cohort (60 DKDs and 116 non-DKDs) [23]. Mean abundance index (MAI) was calculated and analyzed by LEfSe for assessment of different genera. MAI-based ROC curve was applied for identification of subtypes with DN or MN [24]. Area under ROC curve (AUC) values were generated in R (http://www.R-project.org/).

\section{Results:}

\section{Study cohort}

We collected fecal samples from 533 volunteers. After exclusion of 15 DKDs for missing information, 120 patients with DKD, 92 patients with DM and 140 healthy controls were incorporated into discovery phase for bioinformatic analysis. Based on comparison of bacterial community among three groups, microbiota-targeted biomarkers were identified and used to construct disease classifier for differentiation of DKD samples from non-DKD samples (DM and healthy controls). In addition, we designed a small validation phase including $60 \mathrm{DKDs}, 46 \mathrm{DMs}$ and 70 controls to assess diagnosis efficacy of DKD-related classifier. Of all the participates, 60 underwent renal biopsy. Pathological reports, as the diagnostic golden standard of DKD, revealed that 22 were $D N$ while 22 were MN. We further performed dominant flora analysis, marker selection and ROC construction for DN and MN samples (Fig. 1).

\section{Baseline Characteristics Of Participants}


Compared with healthy controls and diabetes, patients with DKD in discovery group showed marked discrepancy in host factors including SBP and $\beta-M G$ etc. $(P<0.001)$. As expected, a significant decrease in estimated glomerular filtration rate (eGFR) and serum level of albumin, while an increase in $24 \mathrm{~h}$ urinary protein ( $24 \mathrm{~h}$-pro) and serum creatinine were observed in DKD vs. DM. All findings demonstrated that a typical clinical characteristic was represented in DKD samples. In comparison of pathological subgroups, significant increase of DM-course and serum creatinine was observed in $D N$ vs. $M N(P<0.01$, Table 1).

\section{OTU-based diversity and composition alteration of gut microbiota in DKD patients}

Delineation of Rarefaction curve, Shannon-Wiener curve, Rank-abundance curve and species accumulation curve integratedly revealed that the estimated OTUs diversity (including richness and evenness) in DKD group was significantly decreased compared to healthy controls, while the diversity of DKD group was higher than that in DM group (Additional file 2: Figure S1). What is more, we used Shannon/Simpson and Chao indices to assess bacterial diversity and richness respectively. We found that alpha diversity in DKDs fell in between DMs and healthy controls (Shannon $=2.77 \mathrm{E}-12$, Simpson $=$ 2.85E-9, Chao = 1.80E-10; Fig. 2a-c; Additional file 3: Table S1). Alpha diversity estimated by Ace and Observed OTU indices was shown in Additional file 2: Figure S2. As exhibited of overlaps in Venn diagram, 1736 of total 2116 OTUs were shared by three groups, while 1822 of 2285 OTUs were shared by DKD and DM. And 58 OTUs were specific for DKD group (Fig. 2d; Additional file 3: Table S2).

To visualize microbial distribution among three groups, we chose PCoA and NMDS analysis based on Bray-Curtis dissimilarity and (un)weighted UniFrac distances to measure beta diversity. The results showed a separating distribution of bacterial community between DKD/DM and Con group mainly in unweighted UniFrac distance (Adonis for PCoA R = 0.0222, $P=0.001$, Fig. 2e; ANOSIM for NMDS, $R=$ 0.0423, $P=0.001$, Fig. $2 g$ ) and weighted UniFrac distance (Adonis for PCoA R = 0.0352, $P=0.001$, Fig. 2f). Other types of beta diversity were shown in Additional file 2: Figure S3. Heatmap in Additional file 2: Figure $\mathrm{S} 4$ was performed to display gut microbiome distribution of top abundant 50 OTUs among three groups and showed symmetrical composition in both DKD and DM (Additional file 3: Table S3). Gut microbiota with mean abundance larger than $0.03 \%$ and $P$ value lower than 0.05 through Wilcoxon test were considered as key OTUs. As a result, 58 OTUs were selected and delineated an apparently different distribution of OTU profile in DKD vs. Con and DM vs. Con. Moreover, differences of microbial OTU distribution between DKD and DM samples were also observed (Additional file 2: Figure S5; Additional file 3: Table S4). Taken together, all findings showed a clear discrimination of microbial diversity or composition in DKD group from diabetes and controls.

\section{Gut microbial communities were distinct among three groups at taxa level}


Average level of microbial composition in patients with DKD, DM and healthy controls was dominated by phyla Firmicutes, Bacteroidetes, Proteobacteria and Verrucomicrobia (all accounting for more than $95 \%$ in three groups, Fig. 3a). Average bacterial communities at the class, order and family level among three groups were shown in Additional file 2: Figure S6. Fecal microbial communities in each sample at the phylum and genus level were presented in Additional file 2: Figure S7. At the genus level, bacterial frameworks of three groups were composed of 103 genera displayed in barplot (Fig. 3b) and heatmap (Additional file 2: Figure S8).

Kruskal-Wallis test was used to compare and identity the difference of gut microbiome at the phylum and genus level. As shown in Fig. 3c and Additional file 3: Table S5, phyla Verrucomicrobia and Actinobacteria were relatively abundant in DKD, followed by DM. While relative abundance of phyla Bacteroidetes and Proteobacteria were increased in Con vs. DKD or DM. Comparison of gut microbiome at the class, order and family level were represented in Additional file 2: Figure S9 and Additional file 3: Table S6, S7, S8. At the genus level, we observed expansion of 33 genera in DKD. Of these discriminatory genera, significant increase of potential pathogens like Peptostreptococcaceae_incertae_sedis,

Erysipelotrichaceae_incertae_sedis, Clostridium_sensu_stricto_1, Streptococcus, Enterococcus and sulphate-reducing bacteria Desulfovibrio were observed in DKD. And genera Streptococcus, Klebsiella and Escherichia-Shigella were enriched in both DKD and DM. Notably, enrichment of beneficial bacteria like Akkermansia, Ruminococcaceae_incertae_sedis, Ruminococcus, Bifidobacterium and Lactobacillus were also observed in DKD group. Whereas, bacteria producing short-chain fatty acids (SCFA) such as Bacteroides, Faecalibacterium,Lachnospira, Blautia, Lachnospiraceae_incertae_sedis and Roseburia were more abundant in healthy controls (Fig. 3d; Additional file 3: Table S9). Additionally, distribution of distinct phyla bacteria between DKD/DM and DKD/Con groups were shown in Additional file 2: Figure S10.

\section{Identification of Major Microbial Markers Associated With Dkd}

LEfSe algorithm was performed on 16S rRNA data to study structural and functional changes associated with gut microbiome in different host states. As shown in Fig. 4a, Additional file 2: Figure S11 and Additional file 3: Table S10, 36 genera with LDA score $>2.0$ and $P$ value $<0.5$ were identified as significantly different gut microbiome. Overall, 14 bacteria were relatively higher in DKD patients than those in DM or controls, while 22 bacteria decreased. Analogous to results above mentioned, most of the significantly different microbiota were harmful bacteria.

Enrichment of KEGG metabolic pathways among DKD patients, diabetes and healthy controls were predicted using PICRUSt. In terms of metabolism module, potential of glycerophospholipid metabolism, biosynthesis of unsaturated fatty acids, bacterial chemotaxis, and others were higher in DKD group. Whereas functional categories of glutathione metabolism, glycerol-lipid metabolism, fatty acid metabolism, lipopolysaccharide biosynthesis, metabolism of aromatic amino acid and others belonging 
to membrane transport and carbohydrate metabolism were significantly increased in DM group. We also observed an increase of metabolic pathways belonging to nucleotide metabolism, metabolism of cofactors and vitamins, fatty acid biosynthesis and metabolism of arginine metabolism in healthy controls (Fig. 4b; Additional file 2: Figure S12; Additional file 3: Table S11).

With five trails of fivefold cross-validation performed on random forest model, 11 optimal OTUs were set as identification biomarkers for 120 DKDs and 232 non-DKDs samples (92 DMs and 140 controls) in discovery phase (Additional file 3: Table S12). Before ROC construction for disease classifier, we first explored correlation between microbial markers and clinical variables in DKD patients. Our unpublished study showed that some clinical parameters were closely related to prognosis of DKD patients. Therefore, we investigated their relationship with microbial biomarkers. Microbiome from $f_{-}$Lachnospiraceae, g_Blautia and g_Faecalibacterium with higher proportion in healthy controls, were correlated positively with hemoglobin level and eGFR, while negatively with serum creatinine, diabetic course and $\beta-M G$. DKDenriched Lactobacillus and Ruminococcaceae_incertae_sedis was positively related to glycosylated hemoglobin, SBP, serum creatinine and 24 h-urinary protein etc. Additionally, the two genera were also negative with eGFR, serum albumin and hemoglobin level (Fig. 4c, Additional file 3: Table S13).

\section{Microbial OTU-based classifier efficiently differentiated DKDs from non-DKDs}

Relationship between cross-validation error rates and number of OTUs indicated that 11 OTU markers could be set as optimal set (Fig. 5a). In discovery phase, a higher average POD value was displayed in DKD group than non-DKD ( $P<0.001$, Fig. 5b; Fig. 4c, Additional file 3: Table S14). POD index-based AUC of training set was $88.12 \%(95 \% \mathrm{Cl} 84.57-91.67 \%$, Fig. $5 \mathrm{C})$. To validate the diagnostic potential of DKD classifier, we introduced a small validation phase (also assigned as testing set) including 60 DKDs and 116 non-DKDs (46 DMs and 70 controls). In validation cohort, average POD value in DKD group was significantly higher than non-DKD ( $P<0.001$, Fig. 5d; Additional file 3: Table S15). The 11 microbial markers could distinguish 60 DKDs from 116 non-DKDs with an area under the ROC curve (AURC) of $79.75 \%$ (95\% $\mathrm{Cl} 73.06-86.43 \%$, Fig. 5 e). These results suggested that microbiota-targeted markers could achieve a high diagnostic efficiency for separating DKD patients from non-DKDs.

\section{Bioinformatic analysis associated with gut microbiome from two pathological sub-types}

Forty-four patients were subjected to renal biopsy and were confirmed as $D N(n=22)$ or $M N(n=22)$. Rank-abundance curve revealed an evenly distributed bacteria in DN or MN samples. PCA analysis showed clear discrimination between-groups (ANOSIM based on weighted UniFrac distance R $=0.1122, \mathrm{P}$ $=0.006$; Additional file 2: Figure S13). Microbial composition of 44 individuals at the order and genus level was shown in Fig. 6a, 6b. At the order level, higher proportion of Pasteurellales in MN patients was observed (Fig. 6c; Additional file 3: Table S16). On looking deeper within bacteria at the genus level, 
relative abundance of anaerobic bacteria including Anaerotruncus, unclutured Christensenellaceae and Coriobacteriaceae were increased in DN than that of MN. While potential pathobionts including Veillonella and Haemophilus were increased in MN (Fig. 6d; Additional file 3: Table S17). These results indicated that these distinct gut microbes might be helpful to determine DN or MN status.

To identify gut microbiome with discriminatory power of classification for DN and MN, LEfSe algorithm was used to perform taxonomic signature analysis between groups. Analogous to results analyzed by Wilcoxon test discussed above, LEfSe identified o_Pasteurellales, $f_{-}$Pasteurellaceae, $f_{-}$Veillonellaceae and several corresponding genera were enriched in $\mathrm{MN}$, while relative abundance of Granulicatella, Butyricimonas and Anaerofilum were more abundant in DN fecal samples (Fig. 6e; Additional file 3: Table S18). We further adopted microbial abundance of genera discussed above as predictive markers and found that the combination of 9 genera except uncultured Ruminococcaceae could separate DN from MN with an AUC of $77.69 \%$ (Fig. 6f).

We also predicted functional changes associated with fecal microbiome communities for DN and MN groups. We found that fatty acid synthesis, methane metabolism and oxidative phosphorylation were relatively increased in DN group compared with $M N$. The $M N$ group exhibited potential of increasing fatty acid metabolism, bacterial toxicity and DNA-related replication and repair (Additional file 2: Figure S14; Additional file 3: Table S19).

\section{Discussion:}

Our study highlighted that gut microbiome-related dysbiosis played a potential role in pathogenesis of DKD patients. We analyzed 16S rRNA sequencing data of fecal microbiome from a large cohort of individuals composed of control, DM and DKD patients. PCOA/NMDS analysis demonstrated a distinct separation of gut microbial profile in DKD/DM compared with healthy controls. Through exploration of microbiome-associated changes in composition and metabolic function, we found that gut microbiome was closely correlated with development of DKD and had a powerful diagnostic potential for DKD classification. Of note, microbiome composition also presented significant differences between pathologic sub-types. To our knowledge, this is the first study focusing on the association between gut microbiome with different pathologic types of renal disease.

Our study had the largest sample size and successfully established disease classifier for DKD and nonDKD (diabetes and healthy controls). More importantly, it was also the first to try to explore microbiomerelated non-invasive test for classifying $\mathrm{DN}$ and $\mathrm{MN}$. New insight in relation to microbiota-targeted biomarkers was proposed and demonstrated in multiple diseases. In analysis of intestinal communities of patients with hepatocellular carcinoma or non-HCC (liver cirrhosis and controls), 30 microbiotatargeted markers were identified and considered as an alternative tool for HCC diagnosis [25]. Previous study carried out a deep shotgun sequencing on fecal samples from Chinese individuals and established a metagenomic linkage group consisting of 6000 microbial markers for diabetes identification [16]. Although possible relationship of microbial alteration with diabetic nephropathy had been first explored in 
Tao's study, gut microbiome was still a relatively new field in human DKD and needed more supports [18]. Thus, we exploited $16 \mathrm{~S}$ ribosomal RNA sequencing data from 120 clinically diagnosed patients with DKD enabling a microbial comparison with $92 \mathrm{DMs}$ and 140 healthy controls. Based on those discriminatory microbial OTUs, DKD-associated classifier was constructed with high AUC (88.12\%) to distinct DKD from non-DKD patients. These results suggested that gut microbial markers were an alternative test for DKD prediction and might be an assistant therapeutic therapy in the future. In clinical practice, clinical judgement alone for DKD was inadequate, which could only achieve a $25 \%$ accuracy in accordance with pathological results [4]. Moreover, renal biopsy could not be performed on the patients with rapidly deteriorating physical condition beyond tolerance, appearance of high serum creatinine or poor coagulation etc. $[5,26]$. We further analyzed all 44 biopsy-proven individuals with DN or MN in the same manner and identified several different genera-level bacteria between two groups. Interestingly, relative abundance of these gut microbiota was applied and achieved an AUC with $77.69 \%$. Successful establishment of DN-related classifier indicated that microbiota-targeted biomarkers might become a non-invasive and inexpensive tool for distinguishing pathologic sub-types of DKD. It also suggested the potential application in differentiating pathologic types of other renal diseases, which revealed a promising area of using microbiota as a substitute test of renal biopsy.

Our findings indicated a moderate degree of bacterial dysbiosis in DKD samples compared with either DM or healthy controls. For example, gut microbiome from DKD samples showed a proportionally increase of opportunistic pathogens, sulphate-reducing bacteria and a proportionally decrease of SCFAproducing bacteria. It was consistent with partial results of Tao's study, in which increased diversity of gut microbiome was also observed in 14 DNs compared with $14 \mathrm{DMs}$, and it was primarily due to the richness of harmful bacteria especially g_Escherichia-Shigella [18]. Opportunistic pathogens could produce various pro-inflammatory components such as lipopolysaccharide (LPS) and peptidoglycans (PGN), trigger host immune response mediated by PGN/TLR2 or LPS/TLR4 pathway and finally facilitate the expression of pro-inflammatory factors (IL-1, IL-6 and TNF-a) $[9,27]$. As reported that bacterial LPS activity was closely correlated with dyslipidemia and chronic inflammation in diabetes-related kidney diseases [28]. PICRUSt analysis showed an enrichment of KEGG pathways like lipid metabolism and bacterial motility in DKD patients. SCFA, energy source of intestinal epithelial cells, played a crucial role in the maintenance of integrity of gut barrier and immune balance $[29,30]$. Decrease of SCFA could weak the protective effect of gut barrier, further promote the translocation of harmful bacteria or endotoxins into circulation or lymphonodus [31]. Moreover, these gut-derived secretions could exacerbate impairment of gut barrier in turn [9]. Correspondingly, SCFA-producing bacteria detected in our study was negatively correlated with serum creatinine. Although there was no statistical significance, negative relations of these beneficial bacteria with $24 \mathrm{~h}$-pro and Ghb were also observed in our study. Further study is needed to explore whether these bacteria are useful for prevention of DKD. Such increase of opportunistic pathogens with commensurate decrease of SCFA-producing bacteria had been reported to be involved in the development of vascular or immune diseases, such as diabetes [16], atherosclerosis disease [11] and autoimmune hepatitis [32]. Thus, it was possible that expansion of harmful bacteria might only be a 
common feature possessed by varieties of disease, instead of disease-specific, which just increased the host's susceptibility to disease.

Additionally, genera Desulfovibrio, the major member of sulphate-reducing bacteria, was observed increased in DKD/DN. Sulphate-producing bacteria was involved in the dissimilation of SCFA [33] and produced cytotoxic byproducts like hydrogen sulfide (H2S) during fermentation [34], which impaired the intestinal barrier and further imbalanced the immune regulation $[35,36]$. Of note, inconsistent with previous observations in vascular disease, proportion of several mucin-degrading bacteria was abnormally increased in DKD/DN. Akkermansia muciniphila, species of Akkermansia, could improve glucose tolerance [37], alleviate fat inflammation in obese individuals through the activation of regulatory T cells mediated by Fox3 [38], and also protected vascular function from atherosclerosis induced by endotoxemia [39]. Abundance of Akkermansia in diabetes was positively correlated with number of goblet cells after metformin intervention [40]. Whether this abnormality could be attributed to effect of metformin use remained to be verified. Ruminococcaceae_incertae_sedis, Ruminococcus, from $f_{-}$Ruminococcaceae, also known as secondary bile acids (SBAs) producing bacteria, played a key role in anti-inflammatory effect mediated by TGR5 bile acid receptor [41].

Higher proportion of pathogens Veillonella and Haemophilus were noticed in MN compared with DN. Ren et al. reported Veillonella disparas the strongest disease-related genera in liver inflammation [32]. However, whether they are related to mechanisms of MN development, especially MN-specific PLA2R (Mtype phospholipase $\mathrm{A} 2$ receptor), still need further investigation.

In addition to aforementioned metabolic changes, functional prediction with respect to aromatic amino acid metabolism such as phenylalanine, tryptophan and tyrosine was enriched in patients with DM. Aromatic amino acids were reported as substrate and fermented by microbiome-related enzymes to produce exogenous endotoxins such as p-cresyl or indoles sulfate, which were involved in vascular sclerosis of renal [42] and heart [43]. Besides, arginine, involved in synthesis of polyamine, was depleted in DKD microbiome. Polyamine, as immunomodulatory metabolites, participated in repair of intestinal mucosa [44]. Above results indicated gut-derived endotoxins might play a crucial role in DKD initiation and development. In a word, the structural and functional alterations of microbiome were associated with DKD pathology and might be able to provide support of microbiota-targeted therapy in the future.

\section{Conclusion}

Taken together, the fecal microbial community was altered markedly in DKD. Our results highlighted the possibility of using gut microbiome as potential biomarkers in diagnosis and differentiation of clinically diagnosed or biopsy-proven DN. For patients who are contraindicated to renal biopsy, gut microbiota test may provide a pivotal alternative. Large-scale studies are required to validate gut microbial markers in cohorts from different population or countries. Further researches focusing on mechanisms of dysbacteriosis-related DN development might help develop novel treatment strategies. 


\section{Abbreviations}

DKD: Diabetic kidney disease; DM: Diabetes; Con: Healthy controls; DN: Diabetic nephropathy; MN: Membranous nephropathy; OUT: Operational taxonomy units; PCoA: Principal coordinate analysis; PCA: Principal component analysis; PC:Pprincipal component; NMDS: Non-metric multidimensional scaling analysis; Adonis: Permutational/Nonparametric multivariate analysis of variance; ANOSIM: Analysis of similarities; LEfSe: Linear discriminate analysis and effect size; CV: Cross-validation; POD: Possibility of disease; ROC: Receiving operational curve; AUC: Area under the curve; Cl: Confidence intervals; DM course: Course of diabetes mellitus; eGFR: Estimated glomerular filtration rate; SBP: Systolic blood pressure; DBP: Diastolic blood pressure; $\mathrm{Hb}$ : Hemoglobin; Ghb: Glycosylated hemoglobin; 24 h-pro: 24 h-urine protein; $\mathrm{Cr}$ : Creatinine; $\beta-M G$ : Beta micro-globulin; Alb: Serum albumin; LYM: Lymphocyte count; GRAN: Neutrophile granulocyte count; T/Cr: Protein creatinine ratio.

\section{Declarations}

\section{Ethics approval and consent to participant}

The study was approved by the Institutional Review Board of the First Affiliated Hospital of Zhengzhou University. All participants had signed written informed consents on enrolment.

\section{Consent for publication}

Not applicable.

\section{Availability of data and materials}

The raw sequencing data generated for current study are available in Sequence Read Archive database under accession number SRPxxxxxx. The datasets supporting the conclusions of this article are included in additional file 3 .

\section{Competing interests}

The authors declare that they have no competing interests.

\section{Funding}

This work was supported by the National Natural Science Foundation of China (Grant Nos. 81873611 and 81700633), Science and Technology Innovation Team of Henan (Grant No. 17IRTSTHN020); Foundation for Leading Personnel of Central Plains of China (Grant No. 194200510006) and National 
Key Research and Development Program of China (2018YFC2000501). The funders designed the study and approved publication of manuscript.

\section{Authors' contributions}

ZZZ and JS designed and supervised the study. ZZZ and JX provided financial support. ZGR and AL helped to design this study. JS, ZGR, SYD, WFZ and FLZ collected samples and clinical data for this project. HYR and $C L$ analyzed and interpreted sequencing data. RXG and YDZ completed the draft of this manuscript. HYW involved in typesetting of this manuscript. JS revised the study. All authors read and approved the final manuscript.

\section{Acknowledgements}

We thank all volunteers denoting samples for our study.

\section{Provenance and peer review}

Not commissioned; externally peer reviewed.

\section{References}

1. Kato M, Natarajan R. Epigenetics and epigenomics in diabetic kidney disease and metabolic memory. Nat Rev Nephrol. 2019;15(6):327-45.

2. Alicic RZ, Rooney MT, Tuttle KR. Diabetic Kidney Disease: Challenges, Progress, and Possibilities. Clin J Am Soc Nephrol. 2017;12(12):2032-45.

3. Anders HJ, Huber TB, Isermann B, Schiffer M. CKD in diabetes: diabetic kidney disease versus nondiabetic kidney disease. Nat Rev Nephrol. 2018;14(6):361-77.

4. Liu S, Guo Q, Han H, Cui P, Liu X, Miao L, Zou H, Sun G. Clinicopathological characteristics of nondiabetic renal disease in patients with type 2 diabetes mellitus in a northeastern Chinese medical center: a retrospective analysis of 273 cases. Int Urol Nephrol. 2016;48(10):1691-8.

5. Clinical competence in percutaneous renal biopsy. Health and Public Policy Committee. American College of Physicians. Ann Intern Med 1988, 108(2):301-303.

6. de Boer IH, Rue TC, Hall YN, Heagerty PJ, Weiss NS, Himmelfarb J. Temporal trends in the prevalence of diabetic kidney disease in the United States. JAMA. 2011;305(24):2532-9.

7. Backhed F, Ley RE, Sonnenburg JL, Peterson DA, Gordon JI. Host-bacterial mutualism in the human intestine. Science. 2005;307(5717):1915-20.

8. Burgueno JF, Abreu MT. Epithelial Toll-like receptors and their role in gut homeostasis and disease. Nat Rev Gastroenterol Hepatol. 2020;17(5):263-78. 
9. Ramezani A, Raj DS. The gut microbiome, kidney disease, and targeted interventions. J Am Soc Nephrol. 2014;25(4):657-70.

10. Karlsson FH, Tremaroli V, Nookaew I, Bergstrom G, Behre CJ, Fagerberg B, Nielsen J, Backhed F. Gut metagenome in European women with normal, impaired and diabetic glucose control. Nature. 2013;498(7452):99-103.

11. Liu H, Chen X, Hu X, Niu H, Tian R, Wang H, Pang H, Jiang L, Qiu B, Chen X, et al. Alterations in the gut microbiome and metabolism with coronary artery disease severity. Microbiome. 2019;7(1):68.

12. Shah NB, Allegretti AS, Nigwekar SU, Kalim S, Zhao S, Lelouvier B, Servant F, Serena G, Thadhani RI, Raj DS, et al. Blood Microbiome Profile in CKD: A Pilot Study. Clin J Am Soc Nephrol. 2019;14(5):692-701.

13. Vaziri ND, Wong J, Pahl M, Piceno YM, Yuan J, DeSantis TZ, Ni Z, Nguyen TH, Andersen GL. Chronic kidney disease alters intestinal microbial flora. Kidney Int. 2013;83(2):308-15.

14. Poesen R, Windey K, Neven E, Kuypers D, De Preter V, Augustijns P, D'Haese P, Evenepoel P, Verbeke K, Meijers B. The Influence of CKD on Colonic Microbial Metabolism. J Am Soc Nephrol. 2016;27(5):1389-99.

15. Crespo-Salgado J, Vehaskari VM, Stewart T, Ferris M, Zhang Q, Wang G, Blanchard EE, Taylor CM, Kallash M, Greenbaum LA, et al. Intestinal microbiota in pediatric patients with end stage renal disease: a Midwest Pediatric Nephrology Consortium study. Microbiome. 2016;4(1):50.

16. Qin J, Li Y, Cai Z, Li S, Zhu J, Zhang F, Liang S, Zhang W, Guan Y, Shen D, et al. A metagenome-wide association study of gut microbiota in type 2 diabetes. Nature. 2012;490(7418):55-60.

17. Zhao L, Zhang F, Ding X, Wu G, Lam YY, Wang X, Fu H, Xue X, Lu C, Ma J, et al. Gut bacteria selectively promoted by dietary fibers alleviate type 2 diabetes. Science. 2018;359(6380):1151-6.

18. Tao S, Li L, Li L, Liu Y, Ren Q, Shi M, Liu J, Jiang J, Ma H, Huang Z, et al. Understanding the gutkidney axis among biopsy-proven diabetic nephropathy, type 2 diabetes mellitus and healthy controls: an analysis of the gut microbiota composition. Acta Diabetol. 2019;56(5):581-92.

19. Kdoqi. KDOQI Clinical Practice Guidelines and Clinical Practice Recommendations for Diabetes and Chronic Kidney Disease. Am J Kidney Dis. 2007;49(2 Suppl 2):12-54.

20. Tervaert TW, Mooyaart AL, Amann K, Cohen AH, Cook HT, Drachenberg CB, Ferrario F, Fogo AB, Haas $M$, de Heer E, et al. Pathologic classification of diabetic nephropathy. J Am Soc Nephrol. 2010;21(4):556-63.

21. Peng W, Huang J, Yang J, Zhang Z, Yu R, Fayyaz S, Zhang S, Qin YH. Integrated 16S rRNA Sequencing, Metagenomics, and Metabolomics to Characterize Gut Microbial Composition, Function, and Fecal Metabolic Phenotype in Non-obese Type 2 Diabetic Goto-Kakizaki Rats. Front Microbiol. 2019;10:3141.

22. Edgar RC. UPARSE: highly accurate OTU sequences from microbial amplicon reads. Nat Methods. 2013;10(10):996-8.

23. Feng Q, Liang S, Jia H, Stadlmayr A, Tang L, Lan Z, Zhang D, Xia H, Xu X, Jie Z, et al. Gut microbiome development along the colorectal adenoma-carcinoma sequence. Nat Commun. 2015;6:6528. 
24. Mazurowski MA, Desjardins A, Malof JM. Imaging descriptors improve the predictive power of survival models for glioblastoma patients. Neuro Oncol. 2013;15(10):1389-94.

25. Ren Z, Li A, Jiang J, Zhou L, Yu Z, Lu H, Xie H, Chen X, Shao L, Zhang R, et al. Gut microbiome analysis as a tool towards targeted non-invasive biomarkers for early hepatocellular carcinoma. Gut. 2019;68(6):1014-23.

26. Fiorentino M, Bolignano D, Tesar V, Pisano A, Biesen WV, Tripepi G, D'Arrigo G, Gesualdo L, Group EEIW. Renal biopsy in patients with diabetes: a pooled meta-analysis of 48 studies. Nephrol Dial Transplant. 2017;32(1):97-110.

27. Liang L, Tan X, Zhou Q, Zhu Y, Tian Y, Yu H, Kijlstra A, Yang P. IL-1beta triggered by peptidoglycan and lipopolysaccharide through TLR2/4 and ROS-NLRP3 inflammasome-dependent pathways is involved in ocular Behcet's disease. Invest Ophthalmol Vis Sci. 2013;54(1):402-14.

28. Lassenius MI, Pietilainen KH, Kaartinen K, Pussinen PJ, Syrjanen J, Forsblom C, Porsti I, Rissanen A, Kaprio J, Mustonen $\mathrm{J}$, et al. Bacterial endotoxin activity in human serum is associated with dyslipidemia, insulin resistance, obesity, and chronic inflammation. Diabetes Care. 2011;34(8):180915.

29. Nylund L, Nermes M, Isolauri E, Salminen S, de Vos WM, Satokari R. Severity of atopic disease inversely correlates with intestinal microbiota diversity and butyrate-producing bacteria. Allergy. 2015;70(2):241-4.

30. Lau WL, Vaziri ND. Gut microbial short-chain fatty acids and the risk of diabetes. Nat Rev Nephrol. 2019;15(7):389-90.

31. Peng L, Li ZR, Green RS, Holzman IR, Lin J. Butyrate enhances the intestinal barrier by facilitating tight junction assembly via activation of AMP-activated protein kinase in Caco-2 cell monolayers. $J$ Nutr. 2009;139(9):1619-25.

32. Wei Y, Li Y, Yan L, Sun C, Miao Q, Wang Q, Xiao X, Lian M, Li B, Chen Y, et al. Alterations of gut microbiome in autoimmune hepatitis. Gut. 2020;69(3):569-77.

33. Muyzer G, Stams AJ. The ecology and biotechnology of sulphate-reducing bacteria. Nat Rev Microbiol. 2008;6(6):441-54.

34. Bhatia M. H2S and Inflammation: An Overview. Handb Exp Pharmacol. 2015;230:165-80.

35. Szabo C. Hydrogen sulphide and its therapeutic potential. Nat Rev Drug Discov. 2007;6(11):917-35.

36. Pitcher MC, Beatty ER, Cummings JH. The contribution of sulphate reducing bacteria and 5aminosalicylic acid to faecal sulphide in patients with ulcerative colitis. Gut. 2000;46(1):64-72.

37. Greer RL, Dong X, Moraes AC, Zielke RA, Fernandes GR, Peremyslova E, Vasquez-Perez S, Schoenborn AA, Gomes EP, Pereira AC, et al. Akkermansia muciniphila mediates negative effects of IFNgamma on glucose metabolism. Nat Commun. 2016;7:13329.

38. Shin NR, Lee JC, Lee HY, Kim MS, Whon TW, Lee MS, Bae JW. An increase in the Akkermansia spp. population induced by metformin treatment improves glucose homeostasis in diet-induced obese mice. Gut. 2014;63(5):727-35. 
39. Li J, Lin S, Vanhoutte PM, Woo CW, Xu A. Akkermansia Muciniphila Protects Against Atherosclerosis by Preventing Metabolic Endotoxemia-Induced Inflammation in Apoe-/- Mice. Circulation. 2016;133(24):2434-46.

40. de la Cuesta-Zuluaga J, Mueller NT, Corrales-Agudelo V, Velasquez-Mejia EP, Carmona JA, Abad JM, Escobar JS. Metformin Is Associated With Higher Relative Abundance of Mucin-Degrading Akkermansia muciniphila and Several Short-Chain Fatty Acid-Producing Microbiota in the Gut. Diabetes Care. 2017;40(1):54-62.

41. Sinha SR, Haileselassie Y, Nguyen LP, Tropini C, Wang M, Becker LS, Sim D, Jarr K, Spear ET, Singh G, et al. Dysbiosis-Induced Secondary Bile Acid Deficiency Promotes Intestinal Inflammation. Cell Host Microbe. 2020;27(4):659-70 e655.

42. Wu IW, Hsu KH, Lee CC, Sun CY, Hsu HJ, Tsai CJ, Tzen CY, Wang YC, Lin CY, Wu MS. p-Cresyl sulphate and indoxyl sulphate predict progression of chronic kidney disease. Nephrol Dial Transplant. 2011;26(3):938-47.

43. Opdebeeck B, Maudsley S, Azmi A, De Mare A, De Leger W, Meijers B, Verhulst A, Evenepoel P, D'Haese PC, Neven E. Indoxyl Sulfate and p-Cresyl Sulfate Promote Vascular Calcification and Associate with Glucose Intolerance. J Am Soc Nephrol. 2019;30(5):751-66.

44. Perez-Cano FJ, Gonzalez-Castro A, Castellote C, Franch A, Castell M. Influence of breast milk polyamines on suckling rat immune system maturation. Dev Comp Immunol. 2010;34(2):210-8.

\section{Figures}


Figure 1

Part 1

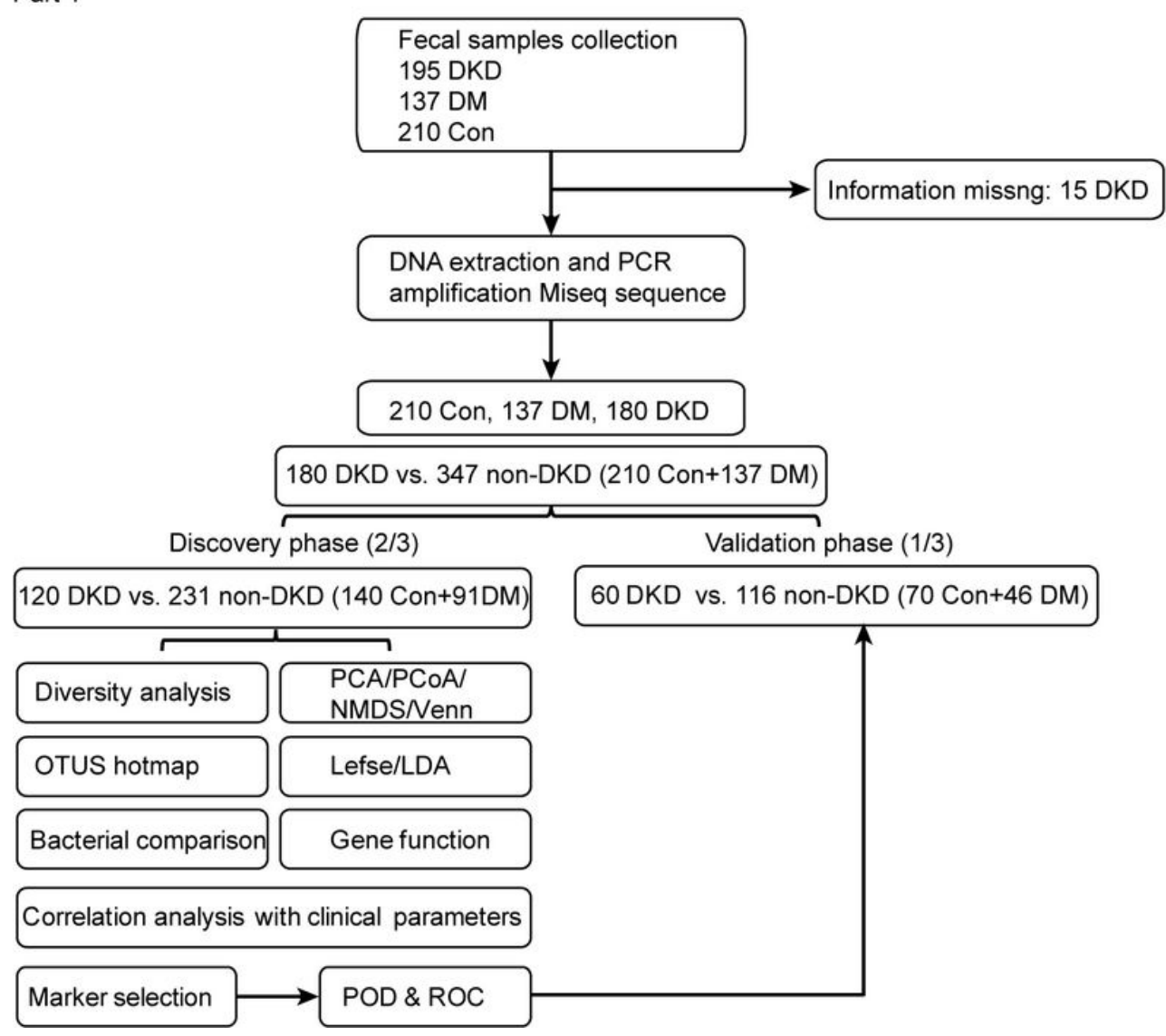

Part 2

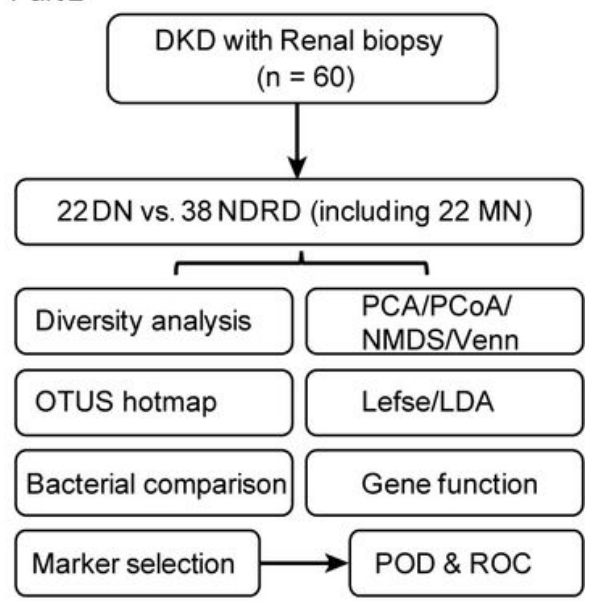

\section{Figure 1}

Experimental flow chart. OTU, operational taxonomy units; DKD, clinically diagnosed diabetic kidney disease; DM, diabetes mellitus; Con, healthy controls; DN, pathologically identified diabetic nephropathy; $\mathrm{MN}$, membranous nephropathy. 

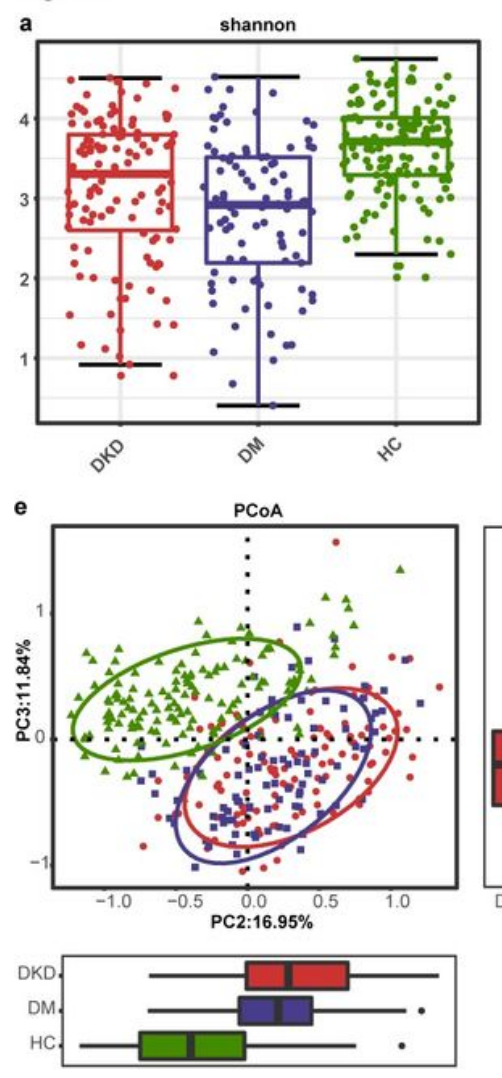
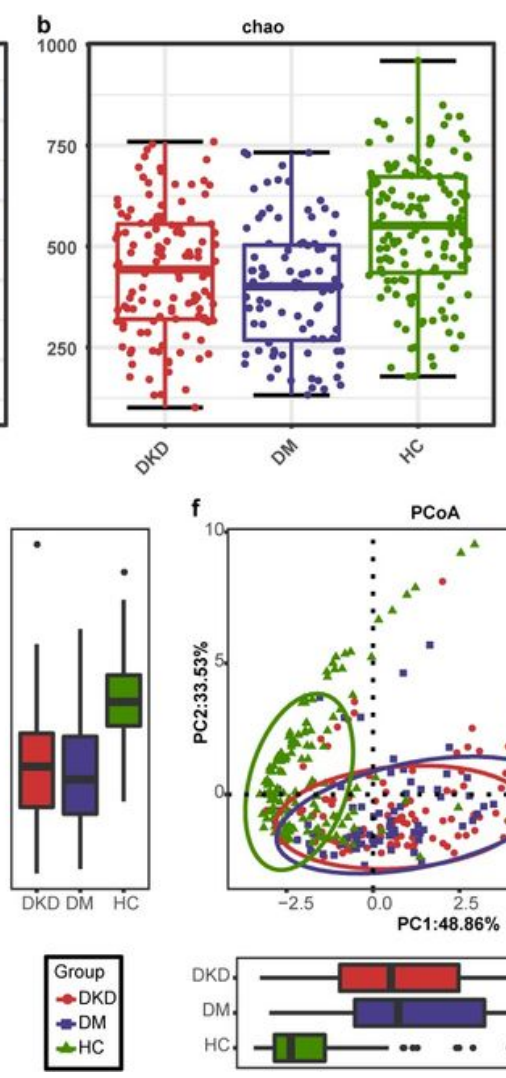

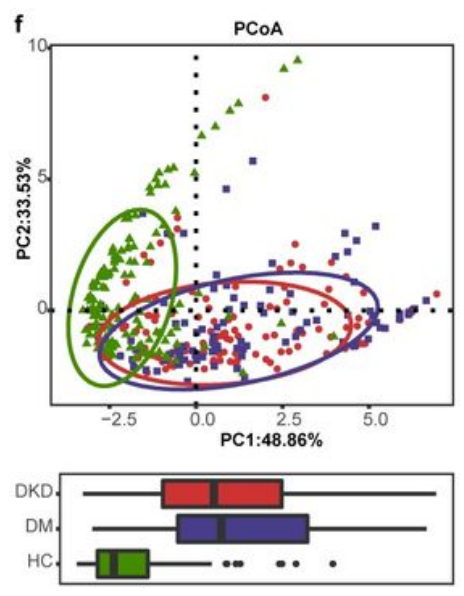

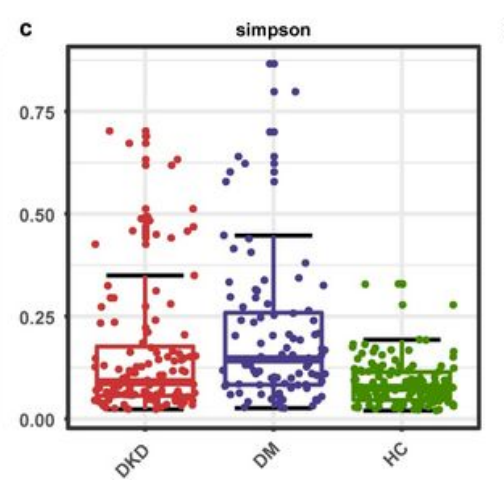
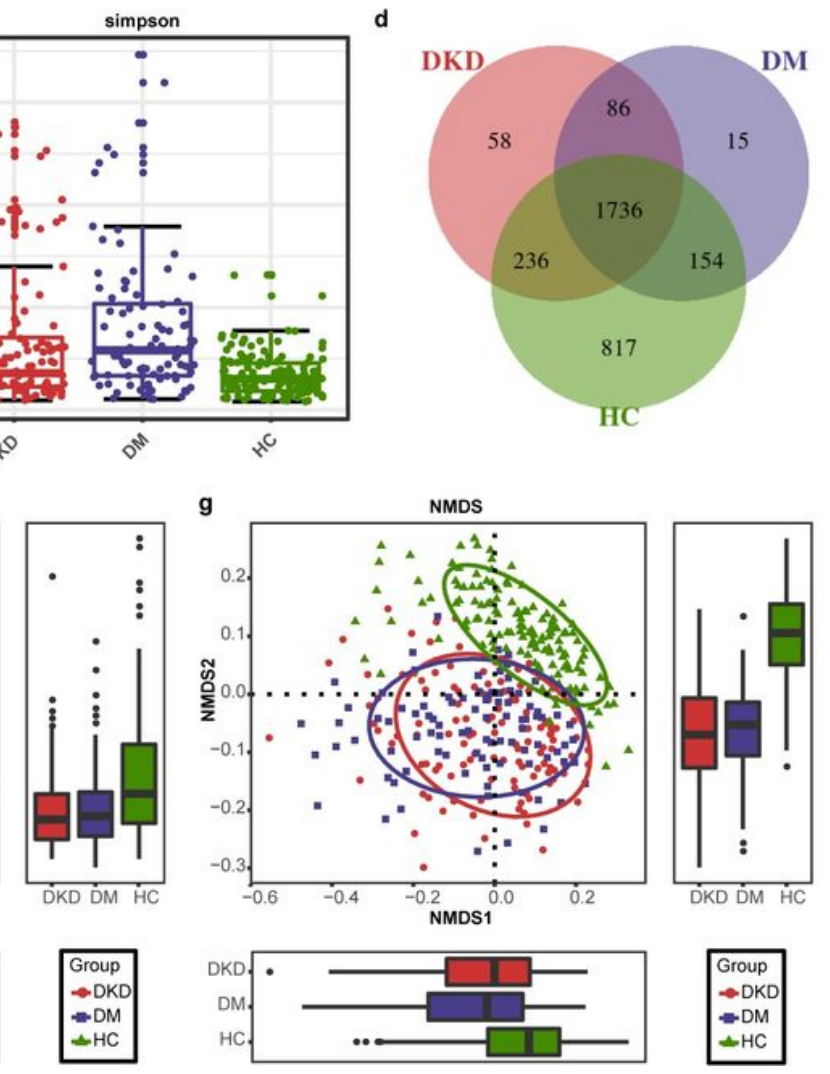

Figure 2

Bacterial diversity in discovery cohort $(D K D=120, D M=92$ and Con=140). a diversity: Bacterial diversity and richness of multi-group comparison were assessed by Shannon/Simpson indices $(a, b)$ and Chao (c) respectively. (d) Venn diagram showed number of observed OTUs among three groups. $\beta$ diversity: PCoA analysis was measured by unweighted UniFrac distance (e) and weighted UniFrac distance (f) at the OTU level. Adonis revealed that weighted analysis taking OTU abundance into account could better reflect the spatial differences among three groups $(R=0.0222$ vs. $0.0353, p=0.001)$. $(g)$ NMDS analysis based on unweighted UniFrac distance (ANOSIM, $R=0.0423, P=0.001$ ). PCoA, principal coordinate analysis; PC, principal component, PC1, PC2 and PC3. NMDS, non-metric multidimensional scaling analysis; Adonis, permutational/nonparametric multivariate analysis of variance; ANOSIM, analysis of similarities. 

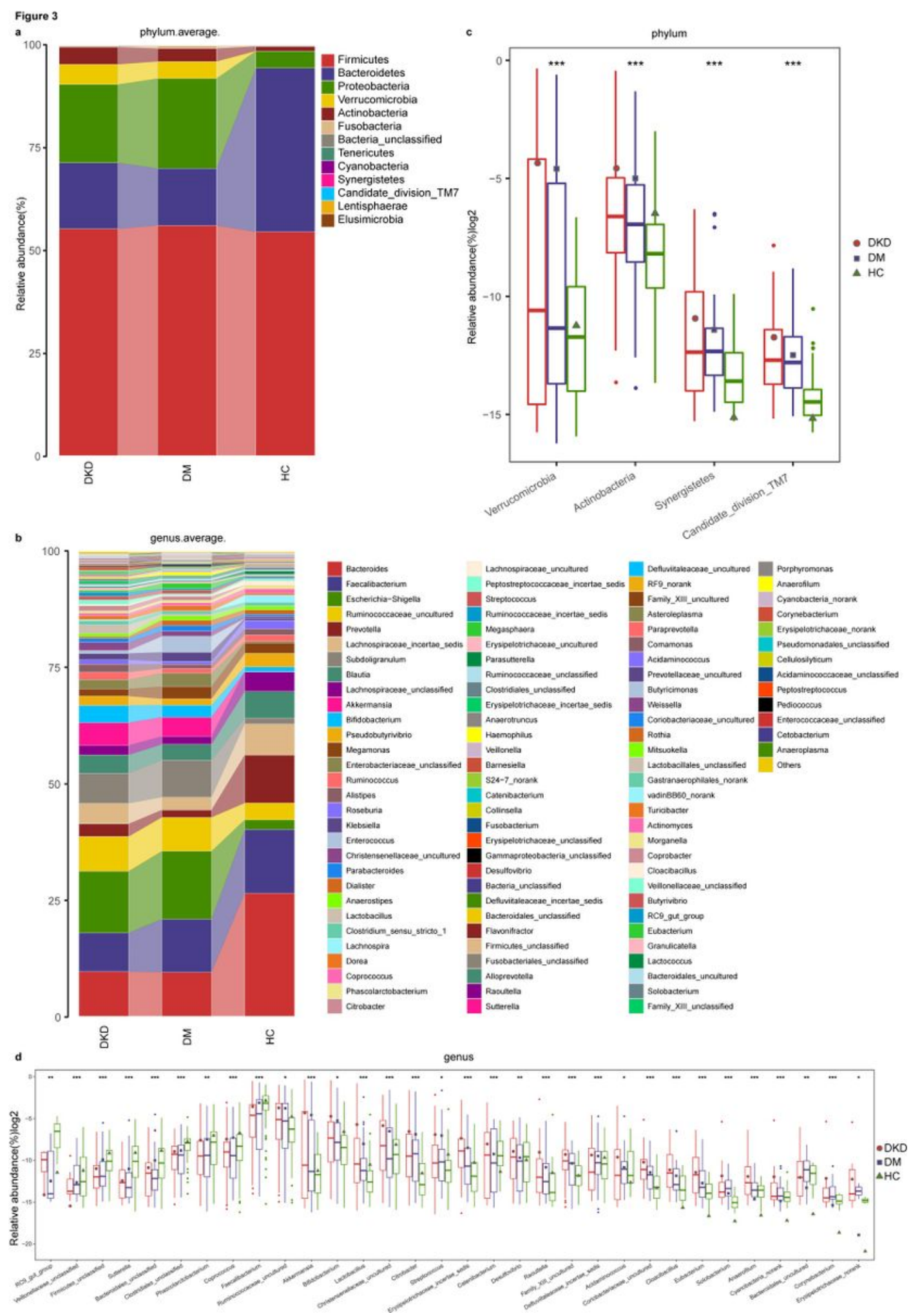

Figure 3

Microbial communities altered among patients with DKD囚DM or healthy controls. In discovery phase, composition of gut microbiome at the phylum (a) and genus (b) level. Kruskal-Wallis rank-sum test was used to compare and identity significantly different bacteria at the phylum (c) or genus (d) level. Only bacteria with gradually increased or decreased abundance among three groups were shown. ${ }^{*} P<0.05$, $\star * P<0.01, * * * P<0.001$. 
Figure
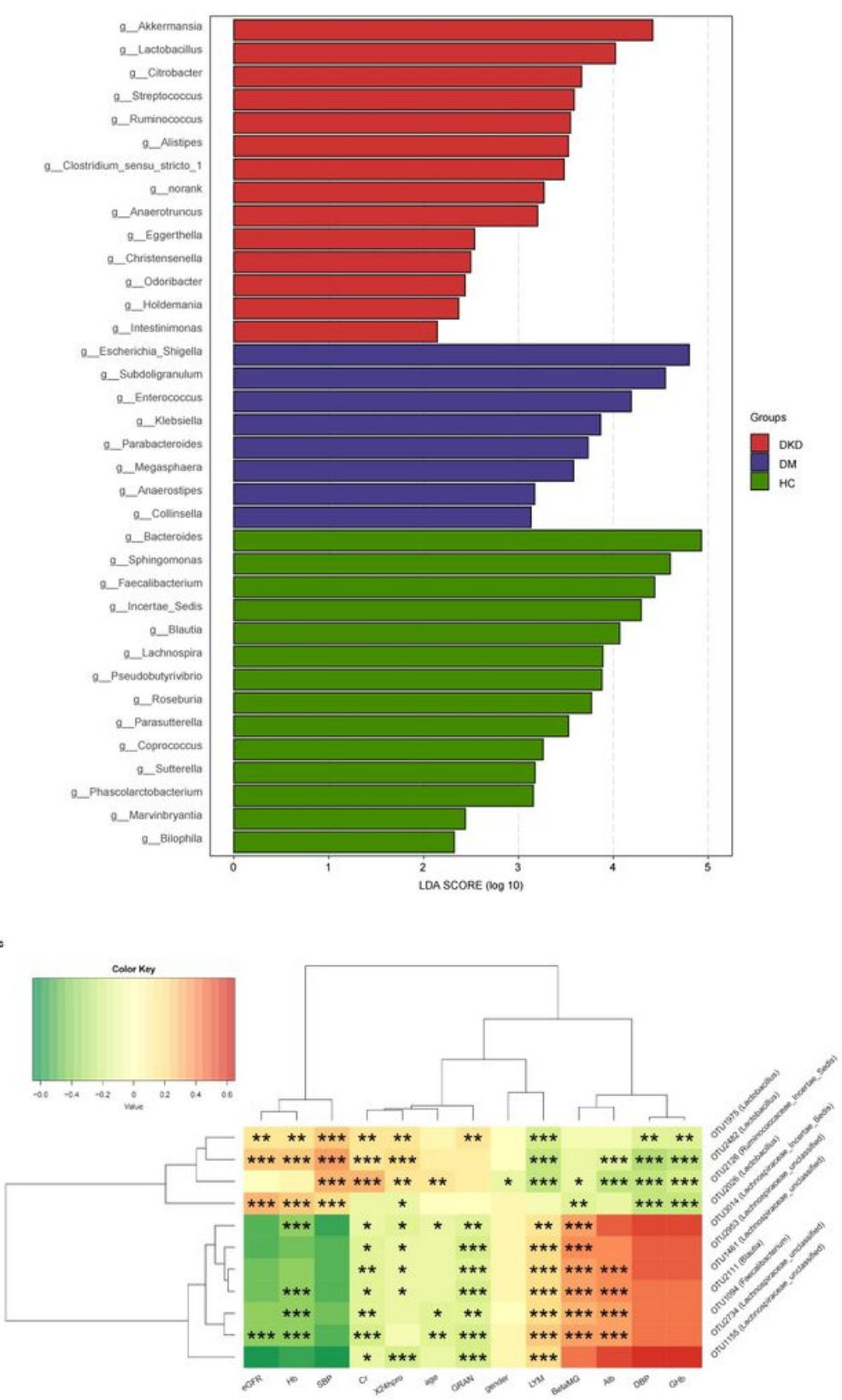

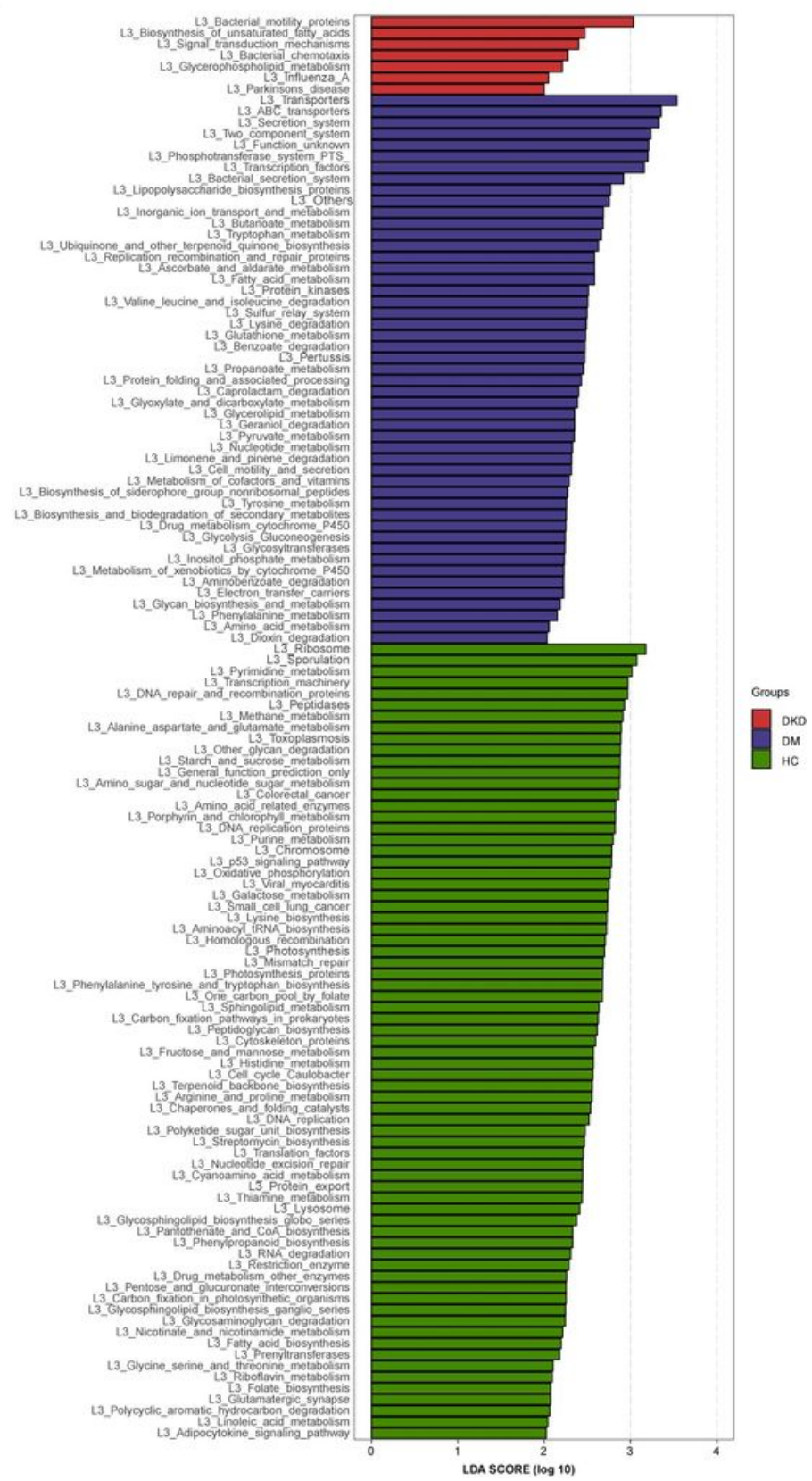

Figure 4

Identification of major microbial markers associated with DKD. (a) Histogram of LDA scores was used to select significant different genera (LDA>2.0 and P<0.05). (b) KEGG pathways at level 3 was indicated by LEfSe analysis with LDA score $\geq 2.0$ and $P \leq 0.05$. (c) Spearman analysis was employed to investigate positive (red) or negative (green) correlations between microbial biomarkers $(n=11)$ and clinical variables. LEfSe, linear discriminate analysis and effect size; spearman rho $>0.1$ or $<-0.1 ; * P<0.01,{ }^{\star *} P<0.01$, $\star * * P<0.001$. 
Figure 5
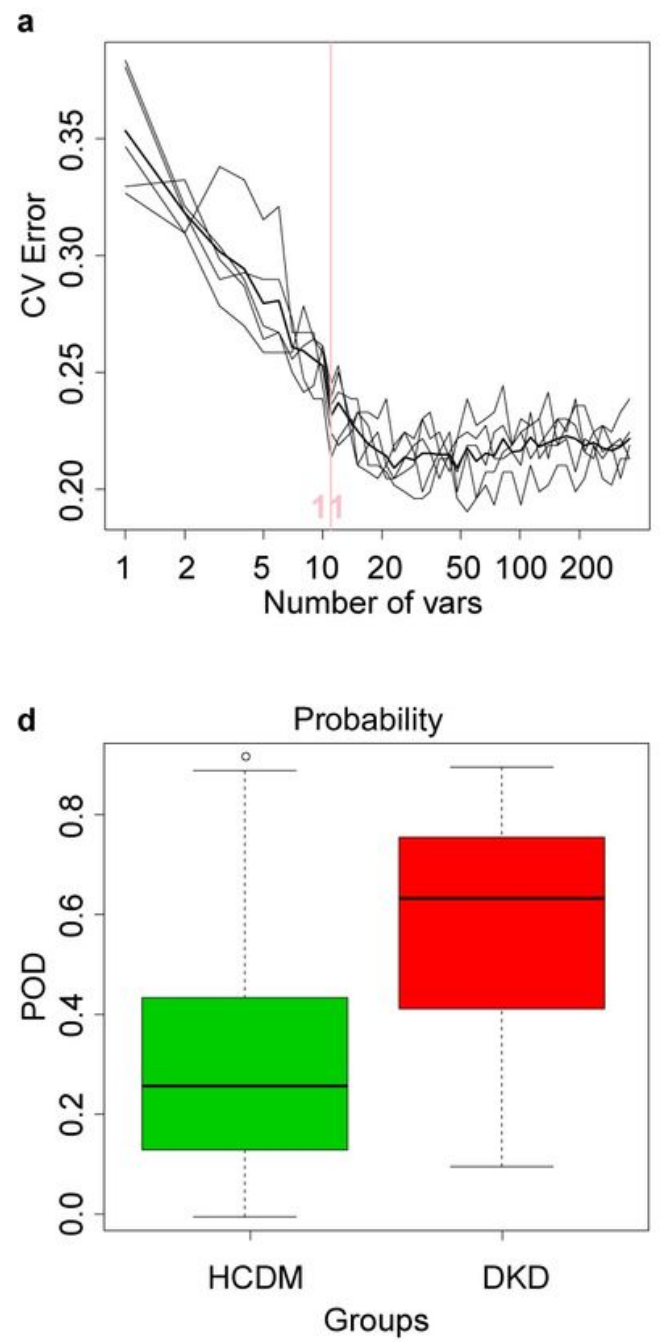
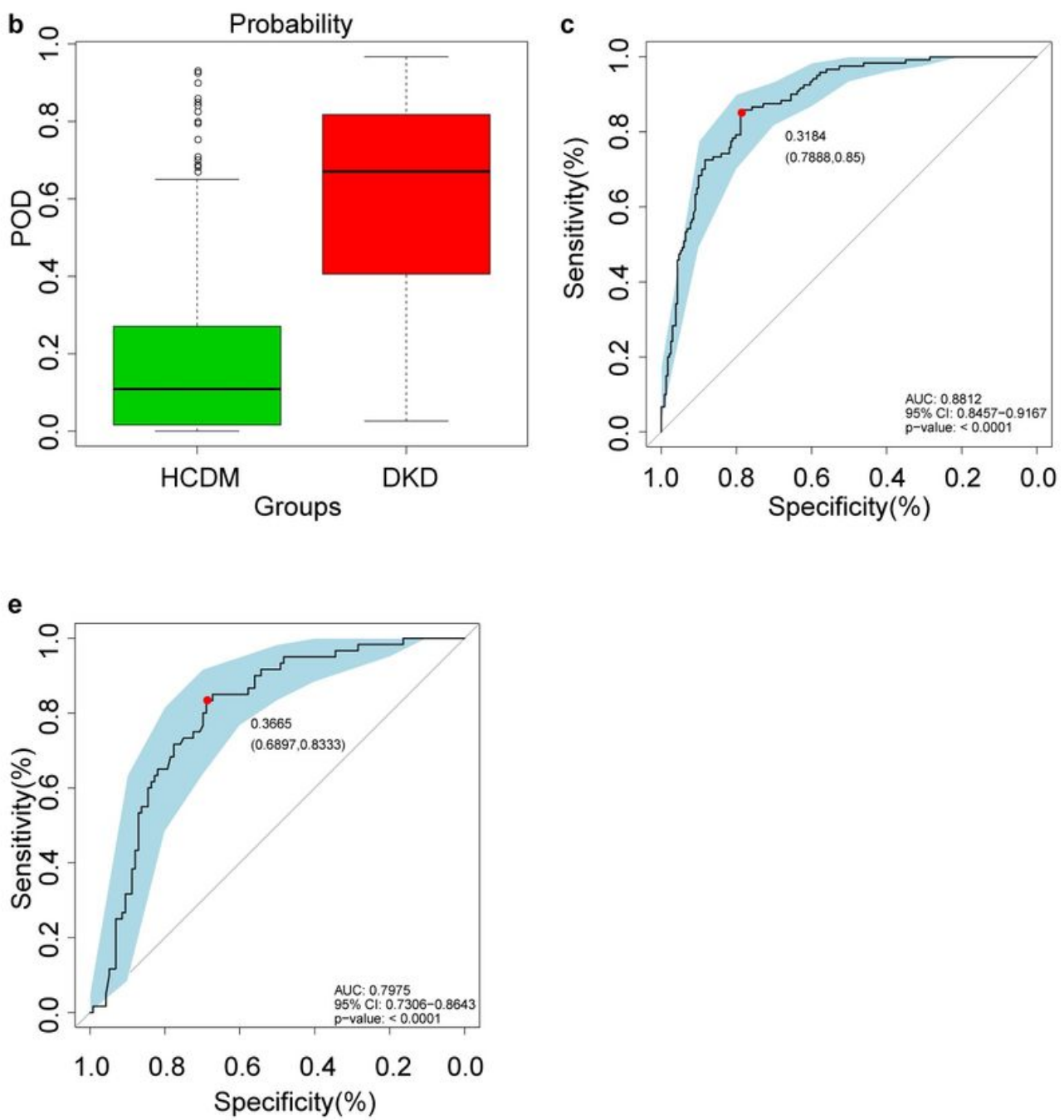

Figure 5

Diagnostic outcomes for DKD and non-DKD in discovery phase and validation phase. (a) Predictive performance of microbial combinations including different key OTU number (computed by 5 -fold crossvalidation and random forest). POD index in discovery phase (b) or validation phase (d) was used to estimate diagnostic efficiency for DKD. Integrating fivefold cross-validation with random forest model, eleven microbial OTUs was identified as optimal diagnostic biomarkers. (c) ROC curve of the DKD-related classifier using 11 microbial biomarkers to distinguish 120 DKDs from 232 non-DKDs. (e) AUC in validation phase verified the predictive power of diagnostic model in 60 DKDs and 116 non-DKDs. Corresponding $95 \% \mathrm{Cl}$ and $\mathrm{P}$ value were shown in graph. CV, cross-validation; POD, possibility of disease; $\mathrm{ROC}$, receiving operational curve; $\mathrm{AUC}$, area under the curve; $\mathrm{Cl}$, confidence intervals. 

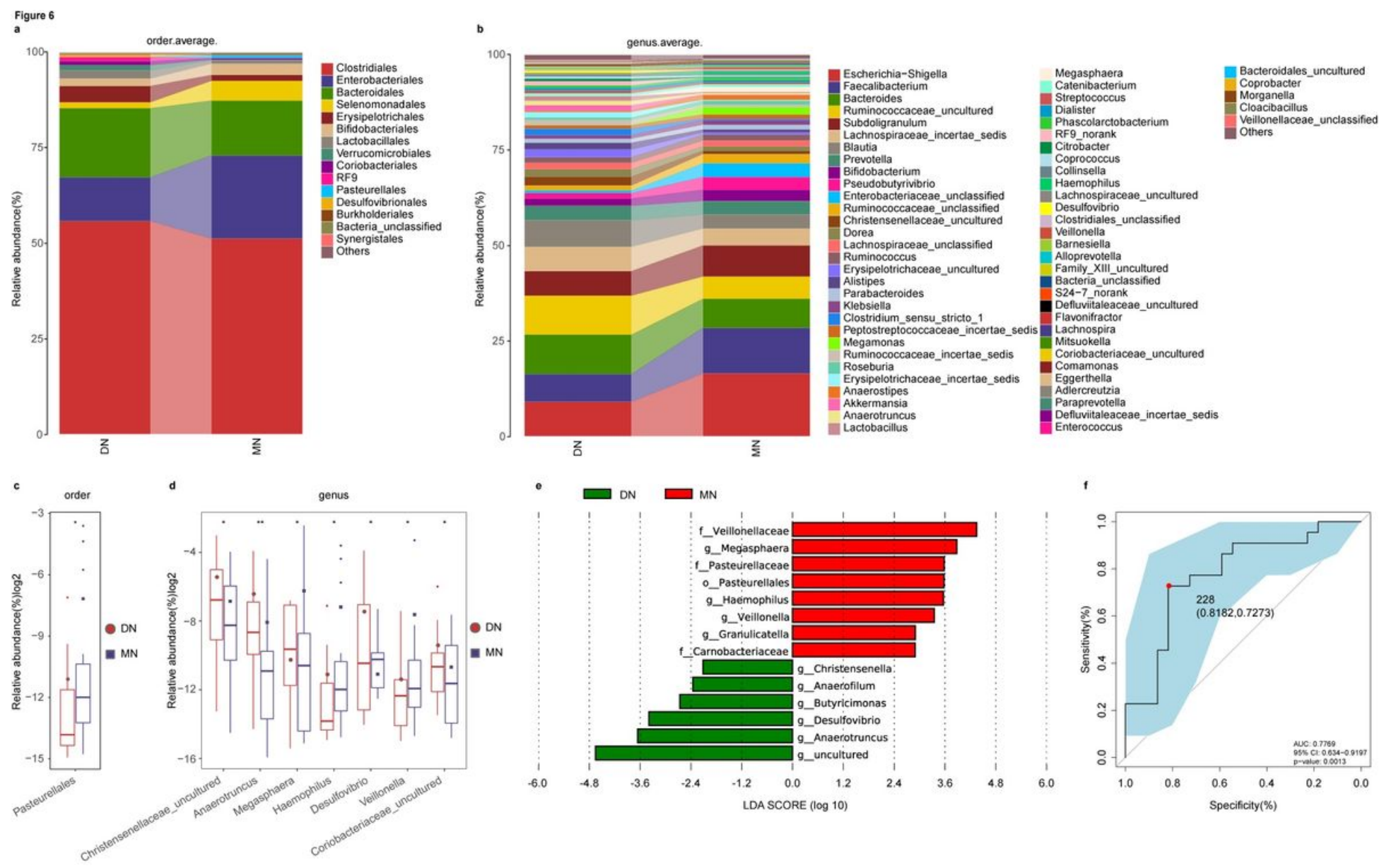

Figure 6

Bioinformatic analysis associated with gut microbiome in two pathologic sub-types of DKD (DN=22 and $M N=22$ ). Comparation of microbial distribution between two groups at the order (a) or genus (b) level. Wilcoxon rank-sum test showed differential microbiome between DN and MN at the order (c), or genus level (d). (e) Gut microbial variations of 9 genera was detected as significantly different using LEfSe analysis (LDA>2.0). (f) ROC curve was constructed to separate biopsy-proven DN from MN. In boxplot, ${ }^{*} p<0.05,{ }^{*} \mathrm{p}<0.005$.

\section{Supplementary Files}

This is a list of supplementary files associated with this preprint. Click to download.

- Additionalfile2.rar

- Additionalfile3.rar

- Additionalfile1.doc 